\title{
Channel Noise is Essential for Perithreshold Oscillations in Entorhinal Stellate Neurons
}

\author{
Alan D. Dorval Jr and John A. White \\ Department of Biomedical Engineering, Center for BioDynamics, Center for Memory and Brain, Boston University, Boston, Massachusetts 02215
}

Previous experimental and computational work (for review, see White et al., 2000) has suggested that channel noise, generated by the stochastic flicker of voltage-gated ion channels, can be a major contributor to electrical membrane noise in neurons. In spiny stellate neurons of the entorhinal cortex, we remove the primary source of channel noise by pharmacologically blocking the native persistent $\mathrm{Na}^{+}$conductance. Via the dynamic-clamp technique (Robinson and Kawai, 1993; Sharp et al., 1993), we then introduce virtual persistent $\mathrm{Na}^{+}$channels into the membranes of the stellate neurons. By altering the mathematical properties of these virtual "knock-ins," we demonstrate that stochastic flicker of persistent $\mathrm{Na}^{+}$channels is necessary for the existence of slow perithreshold oscillations that characterize stellate neurons. Channel noise also alters the ability of stellate neurons to phase lock to weak sinusoidal stimuli. These results provide the first direct demonstration that physiological levels of channel noise can produce qualitative changes in the integrative properties of neurons.

Key words: stochastic dynamics; persistent sodium; theta rhythm; entorhinal; dynamic clamp; excitability

\section{Introduction}

Representations of the external world by the spike trains of individual neurons are typically noisy (Rieke et al., 1997). Although synaptic transmission is often a major contributor to electrical membrane noise in neurons (Mainen and Sejnowski, 1995; Stevens and Zador, 1998), noise generated by the stochastic flicker of voltage-gated ion channels may often make important contributions (White et al., 2000). We studied the effects of channel noise in stellate neurons of the superficial medial entorhinal cortex, a parahippocampal region of the mammalian brain. Stellate neurons generate slow perithreshold oscillations (Alonso and Llinas, 1989) that are believed to contribute to the prominent theta rhythm $(3-12 \mathrm{~Hz})$ seen in the hippocampal formation during active exploration (Buzsaki, 2002). Computational studies of stellate neurons have suggested that channel noise, generated by the stochastic flicker of persistent $\mathrm{Na}^{+}$channels, is necessary to generate perithreshold oscillations over a significant range of parameter space (White et al., 1998, 2000). Our main goal in this study was to examine the effects of channel noise on perithreshold neuronal behavior in an experimental setting.

Studying the effects of channel noise in well controlled experiments is inherently difficult. Channel noise, driven by thermal fluctuations of ion channels between open and closed conductance states, is closely connected with the steady-state and dynamic properties of ion channel activation (Sakmann and Neher, 1995). This connection implies that any attempt to alter channel

Received Aug. 22, 2005; revised Sept. 20, 2005; accepted Sept. 20, 2005

This work was supported by National Institutes of Health Grants R01 MH61604 and R01 NS34425 (J.A.W.). We thank B. Burton and K. Sen for helpful discussions and for critically reviewing a previous version of this manuscript. Correspondence should be addressed to Alan D. Dorval, Department of Biomedical Engineering, Duke University, Box 90281, Durham, NC 27708. E-mail: alan@dorval.us.

D01:10.1523/JNEUROSCI.3557-05.2005

Copyright $\odot 2005$ Society for Neuroscience $\quad$ 0270-6474/05/2510025-04\$15.00/0 noise thermally, pharmacologically, or genetically, will also modify the average gating properties of the channels. We circumvented this problem by pharmacologically blocking native persistent $\mathrm{Na}^{+}$channels and using a custom-designed dynamic-clamp system (Dorval et al., 2001) to knock-in virtual ion channels with user-controllable statistics.

\section{Materials and Methods}

Tissue preparation. All experimental protocols were approved by the Boston University Institutional Animal Care and Use Committee. Horizontal sections of hippocampus and entorhinal cortex were prepared from 15- to 25-d-old Long-Evans rats. After anesthetization with isoflurane and euthanasia, brains were removed and immersed in $0^{\circ} \mathrm{C}$ artificial CSF (ACSF) consisting of the following (in $\mathrm{mm}$ ): $126 \mathrm{NaCl}, 3.0 \mathrm{KCl}, 1.25$ $\mathrm{NaH}_{2} \mathrm{PO}_{4}, 2 \mathrm{MgSO}_{4}, 26 \mathrm{NaHCO}_{3}, 10.0$ D-glucose, and $2.0 \mathrm{CaCl}_{2}$, buffered to $\mathrm{pH} 7.4$ with $95 / 5 \% \mathrm{O}_{2} / \mathrm{CO}_{2}$. Horizontal slices were cut to a thickness of $350 \mu \mathrm{m}$ (Vibratome 1000+; Vibratome, St. Louis, MO). Slices were placed in a $30^{\circ} \mathrm{C}$ ACSF bubble chamber for 30 min before being cooled to room temperature. After resting for $1-7 \mathrm{~h}$, slices were moved to the stage of an infrared, differential interference contrast-equipped microscope (Axioscope 2+; Zeiss, Oberkochen, Germany). Slices were bathed in ACSF with $10 \mu \mathrm{M}$ CNQX, $10 \mu \mathrm{M}$ bicuculline methiodide, and $30 \mu \mathrm{M}$ AP-5 to block ionotropic synaptic activity.

Experimental configuration. Electrodes were drawn on a horizontal puller (P97; Sutter Instruments, Novato, CA) and filled with artificial neuroplasm consisting of the following (in $\mathrm{mM}$ ): $135 \mathrm{~K}$-gluconate, 10.0 HEPES, $5.0 \mathrm{NaCl}, 2.0 \mathrm{MgCl}_{2}, 0.50$ EGTA, 2.0 Tris-ATP, and 0.40 TrisGTP, buffered to $\mathrm{pH} 7.25$ with $\mathrm{KOH}$. Final electrode resistances were between 3.5 and $6.0 \mathrm{M} \Omega$. Electrodes were patched with $\mathrm{G} \Omega$ seals onto layer II entorhinal cortical principal neurons. Suction was applied to break through to the neuroplasm. Stellate neurons were identified as neurons exhibiting $>20 \%$ sag in response to hyperpolarizing current (Alonso and Klink, 1993). Electrophysiological recordings were performed with a current-clamp amplifier (Multiclamp 700A; Molecular Devices, Union City, CA) driven by an analog signal from an $\times 86$ per- 
a

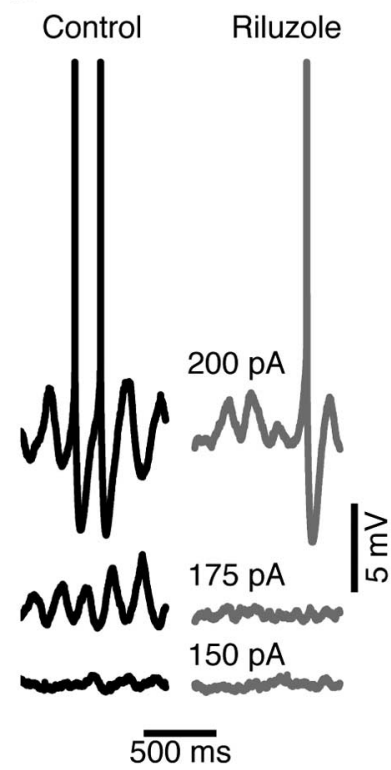

b

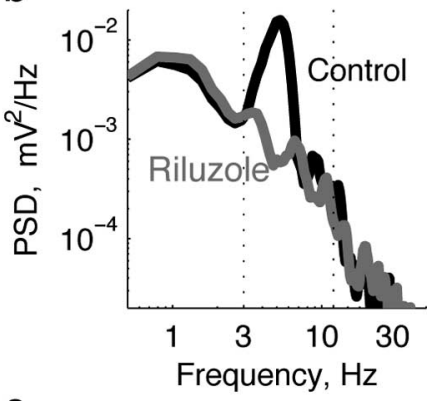

C

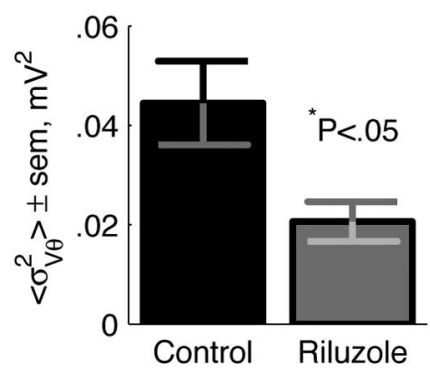

Figure 1. Riluzole abolishes perithreshold membrane oscillations. $\boldsymbol{a}$, Membrane potential traces of an example stellate neuron in response to identified current steps in the absence (left) and presence (right) of $6 \mu \mathrm{m}$ riluzole. $\boldsymbol{b}$, PSDs of the membrane potential in response to perithreshold stimulation (175 pA) in the two conditions. The dotted lines denote the thetafrequency range. $c$, Membrane potential theta power \pm SEM from 12 cells in both conditions. Theta power was defined as the integral of the PSD over theta frequencies identified in $\boldsymbol{b}$. The value of $p$ was calculated from the pairwise $t$ test.

sonal computer running Real-Time Application Interface Linux, and the Real-Time Linux Dynamic Clamp (Dorval et al., 2001).

Conductance implementation. The dynamic-clamp system sampled potential and updated current 13,333 times per second. Persistent $\mathrm{Na}^{+}$ parameters were taken from whole-cell and single-channel patch-clamp work (White et al., 1998; Magistretti and Alonso, 1999; Magistretti et al., 1999). During virtual channel insertion, the persistent $\mathrm{Na}^{+}$current was modeled as follows: $I_{\mathrm{Nap}}=p g_{\mathrm{Nap}}\left(V_{\mathrm{m}}-V_{\mathrm{Na}}\right)$, where $V_{\mathrm{m}}$ is sampled every time step and $g_{\mathrm{Nap}}$ and $V_{\mathrm{Na}}$ are maximum persistent $\mathrm{Na}^{+}$conductance $(3 \mathrm{nS})$ and $\mathrm{Na}^{+}$Nernst potential $(55 \mathrm{mV})$, respectively. The dynamic gating variable $p$ was defined according to the following: $d p / d t=$ $\left(p_{\infty}-p\right) / \tau_{\mathrm{p}}+\eta$, where $\tau_{\mathrm{p}}$ is the persistent $\mathrm{Na}^{+}$time constant $(150 \mu \mathrm{s})$ and $p_{\infty}=0.125 /\left(1+\exp \left[\left(V_{\mathrm{m}}+37.5\right) /-6.5\right]\right)$. The noise variable $\eta$ was set to 0 in the deterministic case, and $p$ was approximated via the exponential Euler method (i.e., solved analytically for the $75 \mu$ s between $V_{m}$ updates). For the stochastic case, the noise term was generated via the Box-Muller stochastic technique (Fox, 1997). The gating variable variance is proportional to the sum of the fractional channel opening ( $\left[p_{\infty}-\right.$ $\left.(1-p)] / \tau_{\mathrm{p}}\right)$ and closing $\left(\left[p-\left(1-p_{\infty}\right)\right] / \tau_{\mathrm{p}}\right)$ rates, and inversely proportional to the number of channels. Thus, $\eta_{k}=W_{k} \operatorname{sqrt}\left[\left(p_{k}+p_{\infty}-2 p_{k}\right.\right.$ $\left.\left.p_{\infty}\right) /\left(2 \tau_{\mathrm{p}} N_{\mathrm{p}}\right)\right]$, where $N_{\mathrm{p}}$ is the number of persistent $\mathrm{Na}^{+}$channels $(1200)$ and subscript $k$ explicitly denotes temporally continuous variables approximated at discrete times. $W_{k}=\cos \left(2 \pi a_{k}\right) \operatorname{sqrt}\left[4 \Delta t \ln \left(b_{k}\right)\right]$ is a zeromean, unit-variance white noise process, where $a_{k}$ and $b_{k}$ are random numbers uniformly distributed between 0 and 1 . The stochastic $p_{k}$ was found as the sum of the exponential Euler method solution and the Box-Muller stochastic term as follows: $p_{k+1}=p_{\infty}-\left(p_{\infty}-p_{k}\right) \exp (-\Delta t)$ $\left.\tau_{\mathrm{p}}\right)+\cos \left(2 \pi a_{k}\right) \operatorname{sqrt}\left[2 \Delta t \ln \left(b_{k}\right)\left(p_{k}+p_{\infty}-2 p_{k} p_{\infty}\right) /\left(\tau_{\mathrm{p}} N_{\mathrm{p}}\right)\right]$.

\section{Results}

We blocked the persistent $\mathrm{Na}^{+}$conductance $\left(g_{\text {Nap }}\right)$ using the neuroprotective agent riluzole (Urbani and Belluzzi, 2000). As expected, bath application of $6 \mu \mathrm{m}$ riluzole greatly reduces the amplitude of $g_{\mathrm{Nap}}$-dependent slow perithreshold oscillations (175 pA) (Fig. 1a). Action potentials, which depend on the tran-

sient $\mathrm{Na}^{+}$conductance, are largely unaffected (200 pA) (Fig. 1a). To quantify the perithreshold oscillation reduction, we calculated the power spectral density (PSD) for both conditions (Fig. 1b), and integrated the PSD over the theta-frequency range. Averaged across 12 cells, riluzole reduced theta-frequency power by $>50 \%$ (Fig. 1c).

With the persistent $\mathrm{Na}^{+}$channels blocked, we used a dynamic-clamp system (Dorval et al., 2001) to knock-in a virtual persistent $\mathrm{Na}^{+}$conductance. In each neuron, we introduced the virtual $g_{\text {Nap }}$, using both deterministic and stochastic models. The deterministic $g_{\text {Nap }}$ was found as the solution of an ordinary differential equation (ODE) of Hodgkin-Huxley form. The stochastic $g_{\text {Nap }}$ was determined by simulating a stochastic differential equation (SDE) constructed using the Langevin approximation that describes the total behavior of a large number of independent, Markov-process-style, persistent $\mathrm{Na}^{+}$channels. In both models, the persistent $\mathrm{Na}^{+}$current was calculated and presented to the stellate neuron in real time with period $75 \mu$ s (Fig. 2a).

Comparison of results under control conditions, with stochastic $g_{\mathrm{Nap}}$, and with deterministic $g_{\mathrm{Nap}}$ show that stochastic knock-ins better replicate control data, especially in the regime of perithreshold oscillations (e.g., $200 \mathrm{pA}$ ) (Fig. 2b). Stellate neurons with a stochastic $g_{\mathrm{Nap}}$ knock-in exhibited prominent thetafrequency oscillations, similar to those seen in control conditions. In contrast, stellate neurons with a deterministic $g_{\text {Nap }}$ knock-in showed no oscillations, similar to behavior seen without any $g_{\text {Nap. }}$. This result is particularly obvious in the power spectra of perithreshold data (Fig. 2c). At a population level, integrated power in the 3-12 $\mathrm{Hz}$ frequency band in the perithreshold regimen is identical for control and stochastic knock-in conditions, but reduced by $>50 \%$ for deterministic knock-ins (Fig. $2 d$ ). The only experimental difference between the two knock-in cases was the variance associated with the stochastic model. Hence, $g_{\text {Nap }}$ variance (i.e., channel flicker) enabled the perithreshold oscillations.

We next explored the effects of channel noise on spiking responses using a stimulus protocol that mimics some aspects of the inputs stellate neurons are likely to receive in vivo: synchronous theta oscillations. For these experiments, we first depolarized stellate neurons with a constant current that induced spikes on one-half of all perithreshold oscillations. To this depolarizing current, we added a sinusoidal component at the natural frequency $\left(f_{\text {nat }}\right)$ of the particular stellate neuron. From trial to trial, we varied the sinusoidal amplitude $\left(I_{\sin }\right)$. For $I_{\sin }<20 \mathrm{pA}$, cells continued to spike on one-half of their oscillations (Fig. $3 a$ ), but the variability of the spike time with respect to the phase of the input depended on $I_{\sin }$ (Fig. $\left.3 b\right)$. For small currents $\left(I_{\sin } \leq 2 \mathrm{pA}\right)$, spike-time variance $\left(\sigma_{t}^{2}\right)$ was large because spikes were temporally splayed, regardless of $g_{\text {Nap }}$ type. For large currents $\left(I_{\text {sin }} \geq 10\right.$ $\mathrm{pA}), \sigma_{t}{ }^{2}$ was small because spikes were phase locked to the input. Most cells exhibited a phase-locking threshold relationship whereby spike-time variance dropped from high to low levels over a small range of $I_{\mathrm{sin}}$. In Figure $3 c$, for example, a phaselocking threshold is observed in the sharp decline in $\sigma_{t}^{2}$ between 5 and $10 \mathrm{pA}$.

To combine data across stellate neurons with different natural frequencies, we multiplied spike-time variance by $12 f_{\text {nat }}^{2}$ and subtracted from 1 to get phase reliability $R_{\varphi}$, a dimensionless measure that ranges from 1 for perfectly phase-locked responses to 0 for maximally dispersed responses. At currents around the phase-locking threshold $\left(2 \mathrm{pA} \leq I_{\sin } \leq 10 \mathrm{pA}\right)$, stochastic $g_{\text {Nap }}$ outperformed deterministic $g_{\mathrm{Nap}}$ at reproducing control spiketime variance and phase reliability. To elaborate, when determin- 
a

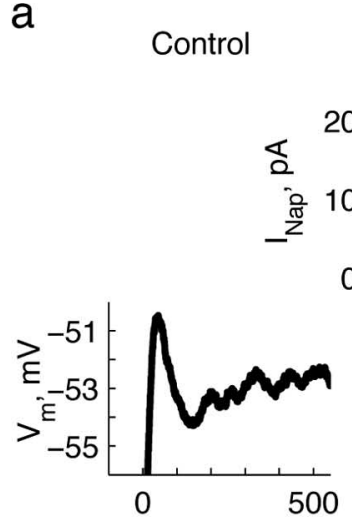

Riluzole \& $\mathrm{SDE} \mathrm{g}_{\mathrm{Nap}}$

b
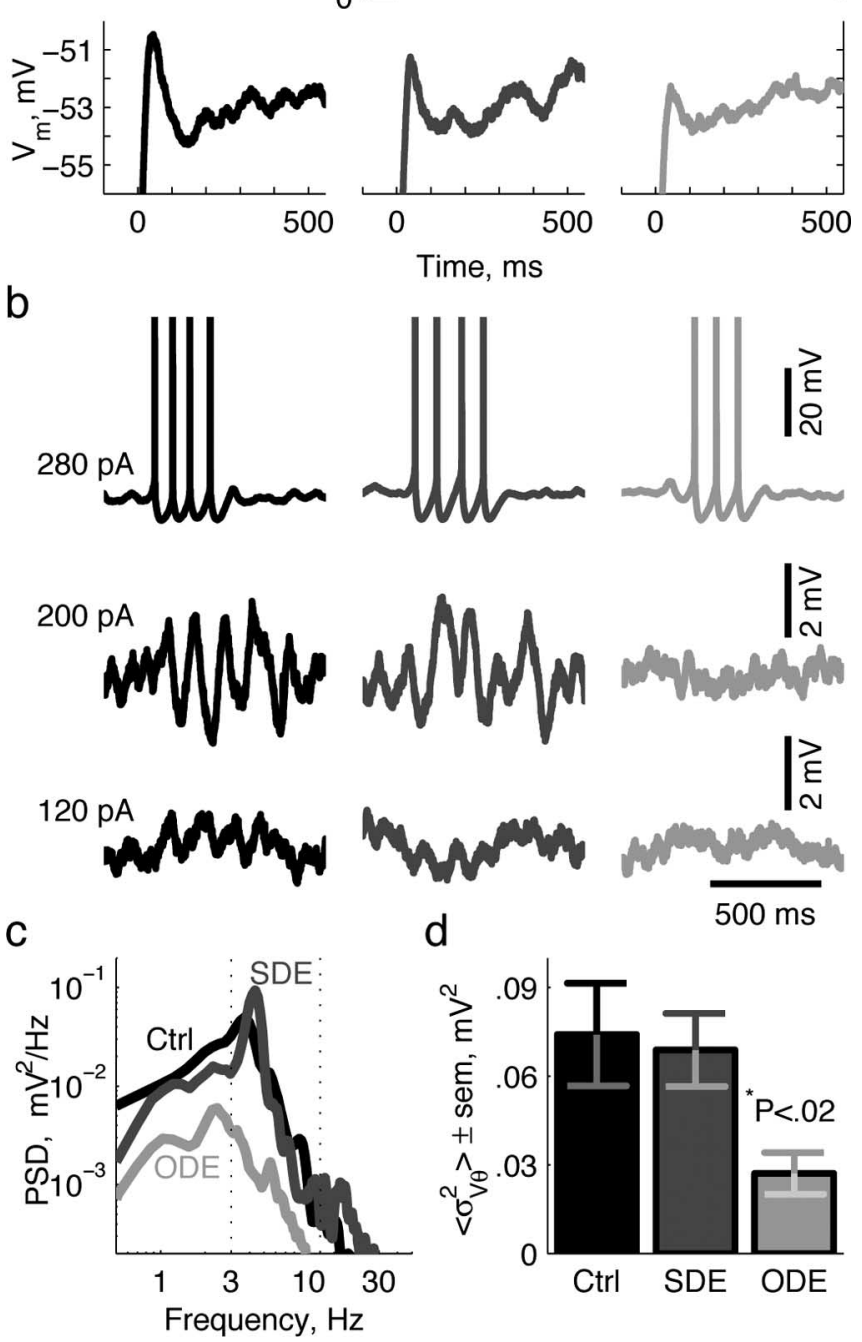

C

Figure 2. Channel noise from persistent $\mathrm{Na}^{+}$channels enables perithreshold oscillations. $\boldsymbol{a}$, Membrane potential traces (bottom) of an example stellate neuron in response to a current step applied at time 0 . Persistent $\mathrm{Na}^{+}$current $\left(I_{\text {Nap }}\right)$ (top), which would have been flowing were it not for the presence of riluzole, was provided to the cell. The ODE model (right) correctly simulated mean $I_{\text {Nap }}$, whereas the SDE model (middle) captured $I_{\text {Nap }}$ mean and variance. Control conditions (left) did not include any virtual $I_{\text {Nap }}, \boldsymbol{b}$, Example traces at three levels of depolarization in the three conditions. c, PSDs of the membrane potential in response to perithreshold stimulation $(200 \mathrm{pA})$ in the three conditions. The dotted lines denote the theta-frequency range. $\boldsymbol{d}$, Membrane potential theta power \pm SEM from 14 cells in all three conditions. Theta power was defined as the integral of the PSD over theta frequencies identified in c. The value of $p$ was calculated from the pairwise $t$ test.

istic $g_{\text {Nap }}$ reduced $\sigma_{t}^{2}$, stochastic $g_{\text {Nap }}$ increased it back toward control levels (5 pA) (Fig. 3c). Likewise, when deterministic $g_{\mathrm{Nap}}$ increased $\sigma_{t}^{2}$, stochastic $g_{\text {Nap }}$ decreased it back toward control levels (10 pA) (Fig. 3c). Similarly, when deterministic $g_{\text {Nap }}$ decreased or increased phase reliability, stochastic $g_{\text {Nap }}$ moved $R_{\varphi}$ back toward control levels (cells 1-6 and 7-12, respectively) (Fig. $3 d)$. Across all cells, the difference in phase reliability, $\Delta R_{\varphi}$, be- a

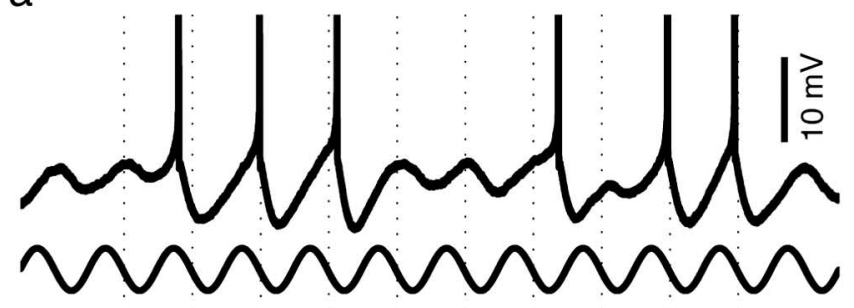

b

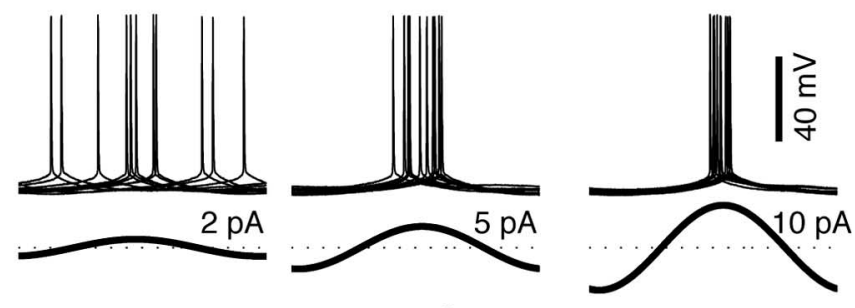

C

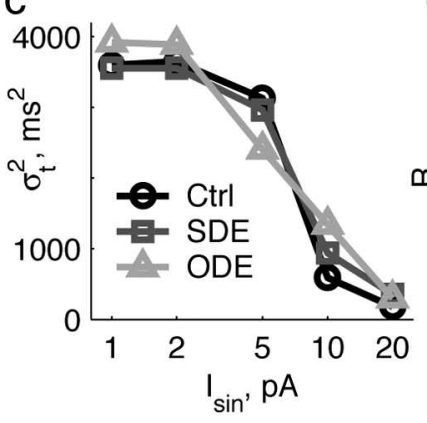

d

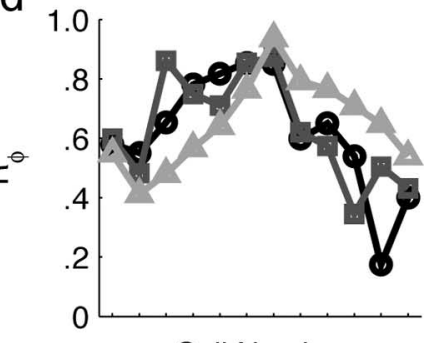

Cell Number

f Below Above e Below Above
Threshold
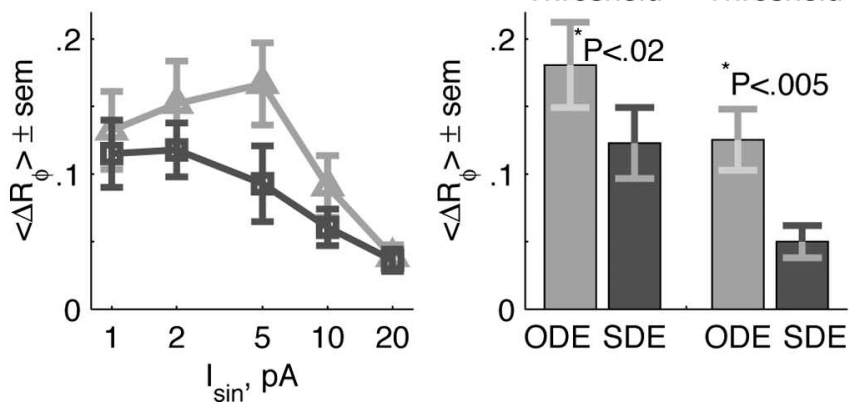

Figure 3. Persistent $\mathrm{Na}^{+}$channel noise sets stellate reliability. $\boldsymbol{a}$, Example membrane potential trace (top) in response to an applied current sine wave ( $3 \mathrm{~Hz} \leq f_{\text {nat }} \leq 9 \mathrm{~Hz}$; bottom). Dashed lines denote the average spike time per cycle, calculated over all spikes from the example trial. $\boldsymbol{b}$, Ten overlaid spikes (top) registered to periodic input (bottom) at three different amplitudes. Note the reciprocal relationship between spike-time variance and input amplitude. c, Spike-time variance for the three conditions, plotted as a function of current amplitude $I_{\text {sin }}$ for an example cell with $f_{\text {nat }}$ equal to $4 \mathrm{~Hz}$. d , Phase reliability for the three conditions of 12 stellate neurons at $I_{\text {sin }}$ equal to $5 \mathrm{pA}$. The cell number is arbitrary. Note that the control and stochastic data are always to the same side of the deterministic data. $\boldsymbol{e}$, The absolute difference in phase reliability between control and experimental conditions as a function of $I_{\sin }$ from all cells. $f$, The absolute difference in phase reliability between control and experimental conditions for the same cells, just below and just above phase-locking threshold, defined as the amplitude at which control spike-time variance dropped below $30 \%$ of its theoretical maximum. The values of $p$ were calculated from the pairwise $t$ test.

tween control and experimental conditions was smaller with stochastic $g_{\text {Nap }}$ than with deterministic $g_{\text {Nap }}$ (Fig. $\left.3 e, f\right)$.

\section{Discussion}

Using dynamic-clamp techniques, we showed that channel noise from persistent $\mathrm{Na}^{+}$channels is necessary for the perithreshold 
oscillations that are the hallmark of spiny stellate neurons in entorhinal cortex. Further, we showed that channel noise affects the ability of stellate neurons to phase lock to weak sinusoidal inputs like those they are likely to receive in vivo during epochs of the theta rhythm (Buzsaki, 2002). Like all knock-in experiments, our technique for replacing native ion channels with virtual counterparts has inaccuracies. We used low concentrations of riluzole that did not block all of the native persistent $\mathrm{Na}^{+}$channels to leave the transient channels intact. We necessarily placed the virtual $g_{\text {Nap }}$ exclusively at the somatic recording site, although the native $g_{\mathrm{Nap}}$ may be located dendritically as well, and we used a fixed number of virtual ion channels rather than fine-tuning this number for each cell. Despite these inaccuracies, stochastic knock-ins reproduced perithreshold oscillations and phase reliability remarkably effectively. In contrast, deterministic knockins never produced perithreshold oscillations and consistently yielded less accurate phase reliability. From these results, we conclude that the stochastic behavior of persistent $\mathrm{Na}^{+}$channels is more important for generating perithreshold oscillations than the inadequacies of our methods mentioned above, and more important than noise from other sources (e.g., flicker in other populations of channels, which we left unblocked). Thus, we provide direct experimental demonstration that channel noise helps establish the dynamical properties of neurons. Our results suggest that microscopic noise contributes to cellular- and systemslevel timing relationships and stimulus-response relationships in entorhinal cortex.

With or without channel noise, stellate neurons exhibit a stable resting potential of around $-65 \mathrm{mV}$ and respond to sufficient depolarization with slow periodic firing (traversals of a limit cycle in the jargon of nonlinear mathematics). The perithreshold oscillations may reflect a spiral trajectory from that limit cycle to rest. Oscillations would occur as the system relaxed from either a noise-induced kick from rest or an action potential. More interestingly, perithreshold oscillations could constitute a weakly repelling unstable limit cycle. Perithreshold oscillations would occur when appropriate persistent $\mathrm{Na}^{+}$channel flicker kicked the system near to that unstable limit cycle from where it could take multiple cycles to relax back to rest. Both interpretations are consistent with previous computational work (White et al., 1998) and the data collected for this study.

Our results are remarkably consistent in that deterministic knock-ins are always inferior to stochastic knock-ins in replicating control results. The most puzzling aspect of our results is that the form of the mismatch in phase reliability differs from case to case. In roughly one-half of the cases, the control and stochastic knock-in data showed reduced phase reliability compared with the deterministic case. These data suggest a model in which realistic levels of channel noise improve the ability of a neuron to follow weak periodic inputs by augmenting perithreshold oscillations and via a noise-enhanced resonance to periodic, perithreshold input. Although we did not explore this noiseenhanced resonance in detail, it is likely related to both stochastic resonance, noise-enhanced detection of subthreshold inputs (Douglas et al., 1993; Collins et al., 1996), and to coherence resonance, noise-enhanced generation of intrinsic periodicity (Pikovsky and Kurths, 1997). In the other one-half of the cases, control and stochastic knock-in data show less reliable phase-locking than in the deterministic case. These data are compatible with a model in which realistic levels of channel noise add jitter to otherwise phase-locked responses.

Perithreshold oscillations, noise-enhanced resonance, and spike-time jitter combine differently across stellate neurons in response to different amplitude periodic inputs. Thus, whether physiological $g_{\text {Nap }}$ variance increases or decreases spike-phase reliability is highly dependent on other variables. However, phase reliability did not correlate with input resistance, capacitance, or any other obvious measure. We hypothesize that phase reliability is sensitive to the relative strengths of $g_{\mathrm{Nap}}$ and other prominent currents (e.g., $I_{\mathrm{h}}$ ), as a function of time and membrane potential. Because of limitations in our ability to space-clamp the cell, the true value of $g_{\text {Nap }}$ is not readily measurable, except in acutely dissociated neurons (White et al., 1998). Future work could examine how the interaction between $g_{\text {Nap }}$ variance and other intrinsic conductances establishes reliability on a cell-by-cell basis.

\section{References}

Alonso A, Klink R (1993) Differential electroresponsiveness of stellate and pyramidal-like cells of medial entorhinal cortex layer II. J Neurophysiol 70:128-143.

Alonso A, Llinas RR (1989) Subthreshold $\mathrm{Na}^{+}$-dependent theta-like rhythmicity in stellate cells of entorhinal cortex layer II. Nature 342:175-177.

Buzsaki G (2002) Theta oscillations in the hippocampus. Neuron 33:325-340.

Collins JJ, Imhoff TT, Grigg P (1996) Noise-enhanced information transmission in rat SA1 cutaneous mechanoreceptors via aperiodic stochastic resonance. J Neurophysiol 76:642-645.

Dorval AD, Christini DJ, White JA (2001) Real-time linux dynamic clamp: a fast and flexible way to construct virtual ion channels in living cells. Ann Biomed Eng 29:897-907.

Douglas JK, Wilkens L, Pantazelou E, Moss F (1993) Noise enhancement of information transfer in crayfish mechanoreceptors by stochastic resonance. Nature 365:337-340.

Fox RF (1997) Stochastic versions of the Hodgkin-Huxley equations. Biophys J 72:2068-2074

Magistretti J, Alonso A (1999) Biophysical properties and slow voltagedependent inactivation of a sustained sodium current in entorhinal cortex layer-II principal neurons: a whole-cell and single-channel study. J Gen Physiol 114:491-509.

Magistretti J, Ragsdale DS, Alonso A (1999) High conductance sustained single-channel activity responsible for the low-threshold persistent $\mathrm{Na}^{+}$ current in entorhinal cortex neurons. J Neurosci 19:7334-7341.

Mainen ZF, Sejnowski TJ (1995) Reliability of spike timing in neocortical neurons. Science 268:1503-1506.

Pikovsky AS, Kurths J (1997) Coherence resonance in a noise-driven excitable system. Phys Rev Lett 78:775-778.

Rieke F, Warland D, de Ruyter van Steveninck R, Bialek W (1997) Spikes: exploring the neural code. Cambridge, MA: MIT.

Robinson HP, Kawai N (1993) Injection of digitally synthesized synaptic conductance transients to measure the integrative properties of neurons. J Neurosci Methods 49:157-165.

Sakmann B, Neher E (1995) Single-channel recording, Ed 2. New York: Plenum.

Sharp AA, O’Neil MB, Abbott LF, Marder E (1993) Dynamic clamp: computer-generated conductances in real neurons. J Neurophysiol 69:992-995.

Stevens CF, Zador AM (1998) Input synchrony and the irregular firing of cortical neurons. Nat Neurosci 1:210-217.

Urbani A, Belluzzi O (2000) Riluzole inhibits the persistent sodium current in mammalian CNS neurons. Eur J Neurosci 12:3567-3574.

White JA, Klink R, Alonso A, Kay AR (1998) Noise from voltage-gated ion channels may influence neuronal dynamics in the entorhinal cortex. J Neurophysiol 80:262-269.

White JA, Rubinstein JT, Kay AR (2000) Channel noise in neurons. Trends Neurosci 23:131-137. 